\section{(6) OPEN ACCESS}

\title{
Sports-related extensor carpi ulnaris pathology: a review of functional anatomy, sports injury and management
}

\author{
Doug Campbell, ${ }^{1}$ Rob Campbell, ${ }^{2}$ Phil O'Connor, ${ }^{3}$ Roger Hawkes ${ }^{4}$
}

\begin{abstract}
${ }^{1}$ Department of Orthopaedic Surgery, Leeds Teaching Hospitals NHS Trust, Leeds, UK ${ }^{2}$ Department of Radiology, Royal Liverpool University Hospital, Liverpool, UK ${ }^{3}$ Leeds Musculoskeletal Biomedical Research Unit (LMBRU), Department of Radiology, Leeds Teaching Hospitals NHS Trust, Leeds, UK ${ }^{4}$ European Tour Performance Institute, European Tour, Surrey, UK
\end{abstract}

\section{Correspondence to} Dr Roger Hawkes, European Tour Performance Institute, European Tour, Wentworth Drive Virginia Water, Surrey GU25 4LX, UK; RAH@wkes.eu

The authors are all members of the European Tour Medical Advisory Board. Dr Hawkes is an Honorary Lecturer at the Institute of Sport, Exercise and Health at UCL in London, UK.

Received 5 July 2013

Revised 21 August 2013

Accepted 26 August 2013

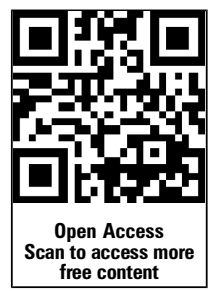

To cite: Campbell $D$ Campbell R, O'Connor $P$, et al. Br J Sports Med 2013:47:1105-1111.

\section{ABSTRACT}

The extensor carpi ulnaris (ECU) muscle plays a key role not only in the active movements of wrist extension and ulnar deviation but also in providing stability to the ulnar side of the wrist. Its position relative to the other structures in the wrist changes with forearm pronation and supination. As such, it must be mobile yet stable. The ECU tendon relies on specific stabilising structures to hold it in the correct positions to perform its different functions. These structures can be injured in a variety of different athletic activities such as tennis, golf and rugby league, yet their injury and disruption is predictable when the mechanics of the ECU and the techniques of the sport are understood. The ECU tendon is also vulnerable to tendon pathologies other than instability. It lies subcutaneously and is easily palpated and visualised with diagnostic ultrasound, allowing early diagnosis and management of its specific conditions. Treatment includes rest, splintage and surgery with each modality having specific indications and recognised outcomes. This review described the functional anatomy in relevant sporting situations and explained how problems occur as well as when and how to intervene.

\section{INTRODUCTION}

The extensor carpi ulnaris (ECU) tendon and its retaining sheath are commonly injured in sport. The anatomy of ECU results in a relatively complex array of injuries seen across a variety of sports. This review describes in detail ECU anatomy, prevalence and mechanisms of sports injury, clinical assessment, imaging findings and the current knowledge base of sports injury management.

\section{Anatomy and function}

ECU is a long thin muscle located on the ulnar aspect of the forearm. It arises from the lateral epicondyle of the distal humerus and inserts at the fifth metacarpal base (figure 1). The muscle's actions vary dependent on the position of forearm rotation.

The ECU tendon passes through a fibro-osseous tunnel (the sixth extensor compartment) as it leaves the forearm, lying within a bony groove on the dorsal surface of the ulna. It is maintained within this groove by a retinaculum and subsheath. The structural integrity of the tendon and the fibro-osseous tunnel are essential for normal wrist mechanics and function.

The retinaculum is an extension of the dorsal retinaculum covering the other five extensor compartments but has no attachment to the ulna. The retinaculum principally prevents bowstringing of the tendon across the wrist during muscle contraction. The ECU subsheath lies deep in the retinaculum and is anchored to the distal ulna.

The ECU muscle provides a variable contribution to wrist flexion and extension dependant on forearm position. In full supination, the ECU tendon lies in a dorsal position relative to the flexion/extension axis of motion, resulting in a greater contribution to true wrist extension. In forearm pronation, the ECU tendon lies more in the palmar and ulnar positions, diminishing its contribution to wrist extension. ${ }^{12}$ Tendon tracking studies have shown that the excursion of ECU during extension diminishes by $60 \%$ when the forearm is positioned in neutral rotation or pronation. ${ }^{3}$

This variation in position of the ECU tendon not only impacts its function, but also its relative stability. In full pronation, the ECU tendon exits the wrist in a straight direction. In forearm supination, the tendon exits the sixth compartment at an angle of approximately $30^{\circ}$ (figure 2). Tension on the ECU retinaculum and subsheath is therefore greater during activities involving forearm supinationespecially when this position is combined with wrist flexion and ulnar deviation. This combination of positions occurs particularly when holding an object tightly and close to the chest (Figure 3).

\section{Pathology}

The ECU tendon disease includes tenosynovitis of the tendon sheath, tendinopathy, tendon disruption and tendon instability. These conditions can occur in isolation or synchronously. Rupture of a normal tendon is very unusual and is only seen in high force, lacerating or penetrating injury.

\section{Tenosynovitis}

Repetitive sporting activity often leads to the development of tenosynovitis. Tendon instability may be a contributing factor. The ECU tendon sheath can be irritated by repetitive flexion and extension of the wrist, particularly in supination, at the point of angulation of the tendon as it exits the fibro-osseous tunnel.

There are reports of stenosing tenosynovitis of ECU, but none that are sports related. ${ }^{4}$ The site of tendon sheath stenosis is unclear and has not been documented. The experience of the authors is that true stenosing tenosynovitis of the ECU tendon sheath is extremely rare.

Inflammatory conditions such as rheumatoid arthritis should be considered in patients with ECU tenosynovitis and no typical predisposing sporting activity. 


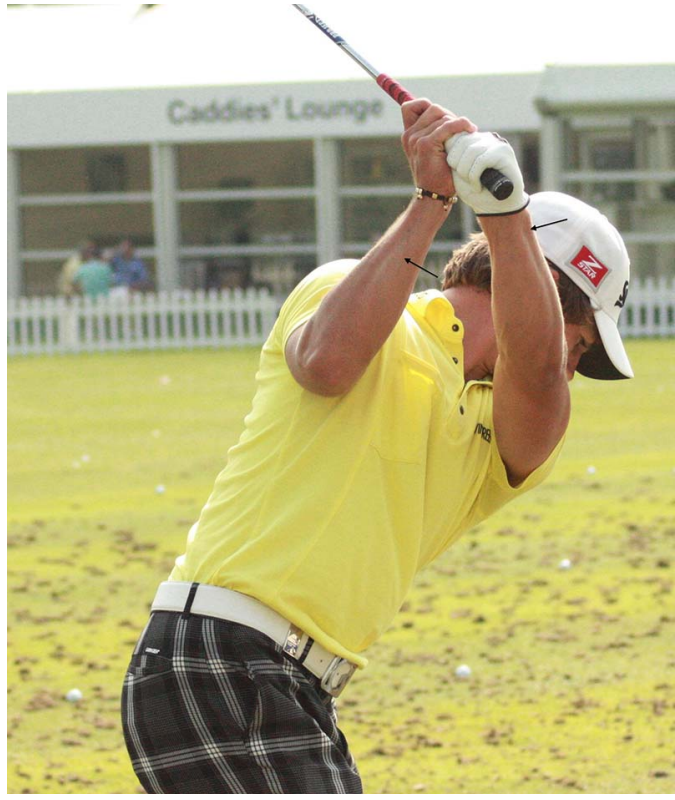

Figure 1 Extensor carpi ulnaris muscle seen on the ulnar side of both of this golfer's wrists.

\section{Tendinopathy}

Tendinopathy is thought to be an adaptive response of the tendon to repetitive stress and/or trauma and may progress through stages of increasing severity. One model describes three different stages of tendinopathy; reactive tendinopathy, tendon disrepair and degenerative tendinopathy, although these occur as a continuum rather than three completely distinct phases. ${ }^{5}$ Reactive tendinopathy typically involves the tendon responding to a rapid increase in loading or from direct trauma. The tendon remains structurally intact and there is a minimal change in collagen integrity. This short-term adaptation to overload thickens the tendon and increases stiffness.

Tendon disrepair occurs with continued excessive loading. The tendon structure begins to change with greater matrix breakdown. There may be an increase in vascularity and neuronal ingrowth. Degenerative tendinopathy is more common in older athletes due to chronic overloading. The tendon becomes less efficient at adaptation to load. Collagen becomes

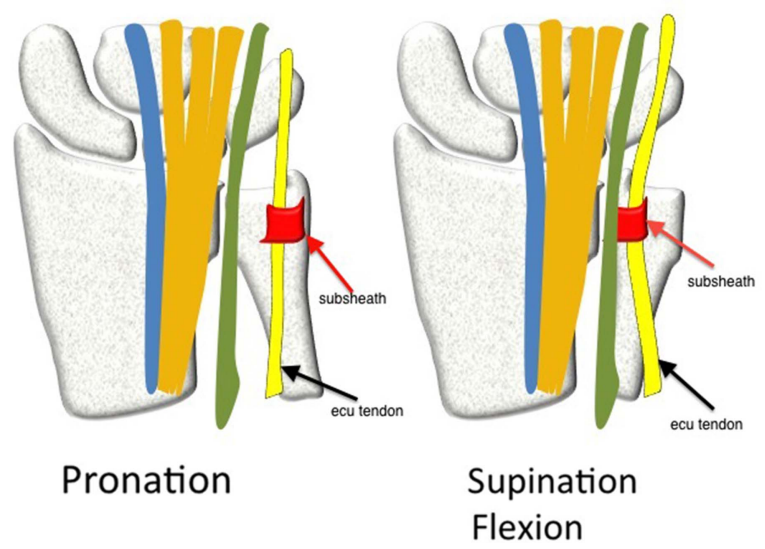

Figure 2 Diagrammatic representation of the change of position of the extensor carpi ulnaris tendon between pronation and supination. There is an angulation of the tendon as it exits the subsheath in supination and flexion.

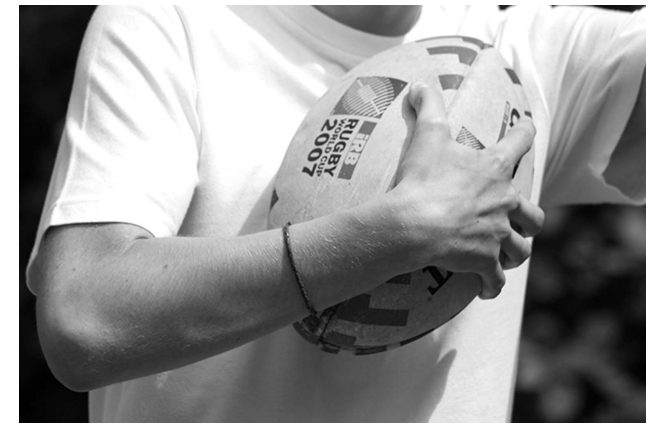

Figure 3 The vulnerable extensor carpi ulnaris tendon in supination, flexion and ulnar deviation in a rugby player.

progressively disorganised with advanced matrix breakdown, which can lead to partial tear and rupture.

Structurally the changes in the tendon can be classified simply as

- Tendinopathy

- Partial rupture

- Complete rupture

- Avulsion/insertion tendinopathy

Tendon instability

There is a spectrum of tendon instability ranging from minor subluxation of ECU during rotational movements to frank dislocation and forearm locking. Instability is the result of disruption or dysfunction of the ECU subsheath. Such acute ruptures usually occur as single traumatic events.

The ECU tendon is vulnerable to instability when it contracts to stabilise the ulnar side of the wrist. The retaining structures are at particular risk of acute injury when the wrist is moving between pronation and supination, while fixed in flexion and ulnar deviation. Less commonly, the subsheath can rupture as a result of repetitive stress.

Disruption of the subsheath occurs at three main sites (figure 4).

1. Periosteal stripping on ulnar wall

2. Radial tear

3. Ulnar tear

The main difficulty with assessing tendon instability is the large variation of movement seen in asymptomatic volunteers. ${ }^{7}$

\section{Sport specific ECU injuries}

The ECU pathology has been most frequently reported in tennis and golf, although injuries to the ECU stabilising structures also occur in certain high impact contact sports, such as rugby league. Sports that risk ECU problems share common features, either loading of the wrist when the ECU is in a vulnerable position that is wrist flexion during supination and ulnar deviation or sudden lateral force applied to the wrist when the tendon is engaged in strong isometric contraction. Reports rarely differentiate between these clinical entities with the overall incidence of wrist injury being reported as up to $8.9 \%$ of all reported sports injuries. 8

\section{Tennis}

A retrospective study of 50 professional tennis players over a 10-year period described 28 individuals with ECU problems. ${ }^{9}$ This was extrapolated to a prevalence of 1 case/18 players/year. Men were more frequently affected with $42 \%$ of all patients having ECU instability, $50 \%$ had tenosynovitis or tendinopathy 

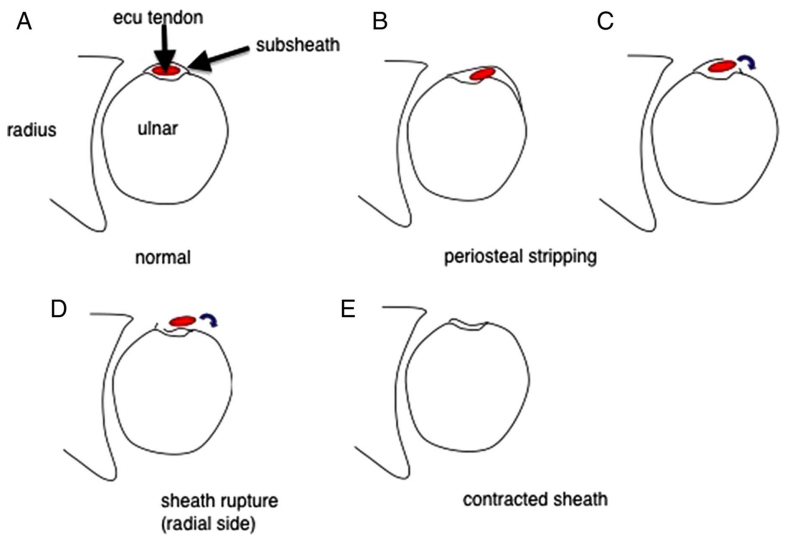

Figure 4 (Subsheath disruption) The types of subsheath rupture comprise (A) normal, (B) periosteal stripping resulting in a false sheath, (C) fibro-osseous sheath rupture at the ulnar side, (D) fibro-osseous sheath rupture at the radial side and (E) a contracted but intact fibro-osseous sheath.

and $8 \%$ presented with complete tendon rupture. Acute injuries typically occur from a double-handed backhand stroke, as the dominant hand moves forcefully and rapidly from pronation to supination to impart spin on the tennis ball. Players presented with the sudden onset of ulnar-sided pain that prevented further play. Symptoms usually resolved following a period of rest, but recurred when attempting to play top spin shots with rapid forearm rotation.

A second group of tennis players described a more gradual onset of ulnar-sided wrist ache that did not interrupt play. This is likely to reflect a tendinopathy since there were no acute signs of tendon subluxation. Two players in this study sustained a complete rupture of the ECU tendon. They described a fluctuating course of chronic symptoms following an initial acute episode, temporarily improved by cessation of precipitating activity. Interestingly, both players had received steroid injections in the weeks prior to the tendon rupture event. Complete tendon rupture did not impact everyday activities, but neitherwere they able to perform a double-handed backhand again due to a lack of power. Therefore, ECU tendon rupture should be regarded as a career threatening injury in such cases.

The style of a tennis player (baseline player or 'serve and volley' player) can also have an impact on the risk (and type) of ECU problems.

The baseline player tends to employ the semi-Western grip, in which the racquet is gripped with the forearm in full supination, so that a full range of $180^{\circ}$ of forearm rotation is available at impact in order to impart as much top spin on the ball as possible.

\section{Golf}

The wrist is a frequent site of injury in amateur as well as professional golfers, although injuries involving the ECU tendon specifically have been poorly documented. The precise incidence and prevalence in golf is unclear. Most published reports are questionnaire surveys undertaken in heterogeneous golfing populations with very low response rates and retrospective in nature. $^{10}$

A survey of the entire competitive field of 153 professional golfers during the 2009 European PGA Tour reported an incidence of wrist injury in 30\% (in production at BJSM). Twelve cases affected ECU (8\%), eight of these were inflammatory problems of the tendon and tendon sheath. There were four cases of ECU subluxation, three of which occurred acutely after a traumatic episode. There was one case of bilateral constitutional sheath laxity.

All cases of traumatic ECU subluxation occurred in the 'leading' wrist (the wrist that faces the target). The 'leading' wrist moves from radial deviation to a neutral position at impact. At this point, the momentum of the golf club is trying to force the 'leading' wrist into ulnar deviation. The ECU tendon contracts isometrically to counteract this effect as the club is brought through impact to the end of the golf swing. If the club strikes a hard object on the ground at impact, the momentum of the swing is suddenly interrupted. The upper body and upper limbs continue to move forwards, while the golf club (and hands) are effectively 'left behind'. This creates a 'traumatic hinge' into the radial deviation of the 'leading' hand. This force combined with the strong isometric contraction of the ECU muscle can result in failure of the subsheath with subsequent subluxation of the ECU tendon. This is perceived as a 'popping' or 'tearing' sensation on the ulnar side of the wrist that is usually sudden and painful.

The remaining group of tour golfers reported less dramatic symptoms diagnosed as ECU tendinopathy. This was associated with the use of hard practice mats or playing off excessively hard ground.

Another questionnaire survey was undertaken on the Ladies European PGA Tour, and included 104 professional golfers (response rate $>90 \%$ ) (John Stanley, personal communication). There was an incidence of wrist injury of 54\%. No information on specific location of symptoms or diagnosis was given, although the 'leading' hand was involved three times more frequently. It is likely that the ECU tendon disease represents a significant proportion of these cases.

\section{Rugby league}

Unpublished data of ECU injuries sustained over a 5-year period in seven English Rugby Super Leagues recorded six acute ECU subsheath injuries, all of which resulted in tendon subluxation. Half of these required surgical repairs. This equates to an incidence of 1 injury/60 players/year.

In this sport, the ball is retained during tackling. Players are trained to hold the ball firmly into their chest as they enter contact. One arm is favoured for this function, (the 'ball carrying' arm). The other arm is used to fend off opponents.

When clutching the ball, the forearm is in maximal supination, with the wrist in flexion and ulnar deviation. The 
isometrically contracting ECU tendon can be clearly seen in images of this technique (figure 3). A sudden increase in the isometric force within the ECU as the player attempts to grip the ball more tightly when entering contact, can result in a traumatic tear of the subsheath with acute subluxation of the ECU tendon in an ulnar direction. This is felt as a painful 'snap' at the moment of impact.

\section{Clinical assessment}

An accurate clinical history and assessment is essential for diagnosis of ECU tendon disorders. There are many other causes of ulnar-sided wrist pain and the area or site of discomfort requires accurate localisation by the patient and examiner.

The timing of onset of symptoms discriminates between acute and chronic causes. Mechanical symptoms at the moment of onset are also common descriptors in this condition. Patients will use words such as 'snap', 'pop' or 'tear' in an acute sheath disruption.

In some cases, episodes of tendon subluxation are excruciatingly painful. In others the subluxation may be entirely asymptomatic and may be easily reproduced by the patient. The subluxing 'snap' of the tendon as it leaves its natural groove on the dorsal surface of the distal ulna can only occur under active contraction of the ECU. Passive movements will not produce this characteristic sign because the tendon is not under sufficient tension to produce the subluxation. Active supination with the affected wrist held tightly in maximal flexion and ulnar deviation will produce often visible subluxation of the tendon.

Demonstrable subluxation that is clear and unambiguous is one end of the spectrum of clinical abnormality. Other clinical signs of tendon pathology may be much more subtle and require careful clinical examination.

Palpation along the length of the ECU tendon (starting distally at its insertion into the base of the fifth metacarpal to ensure palpation of the correct structure) will reveal tenderness accurately localised to that structure. Pain on resisted active extension with ulnar deviation is pathognomic of an ECU condition. Weakness is frequently associated with pain. Painless weakness is likely to represent a complete rupture of the ECU tendon.

In stable ECU tendinopathy, it is more usual to describe an 'area' of discomfort or ache, rather than a specific and accurately described 'point' of pain. In these circumstances, other causes of ulnar-sided wrist pain must be considered. It is common for an individual with stable ECU tendinopathy to describe a growing awareness of functional disturbance and pain.

Patients with active tendinopathy and tenosynovitis of the ECU will often have a vague and subtle swelling over the dorsoulnar aspect of the affected wrist. The pain has two components-a constant dull aching sensation on the dorsoulnar aspect of the affected wrist and a sudden searing longitudinal pain felt along the course of the distal ECU tendon on active contraction of the muscle.

The ECU Synergy Test, ${ }^{11}$ has been shown to be sensitive as well as specific for the diagnosis of ECU tendinosis. It is performed with the patient's elbow resting on the examination table, flexed at $90^{\circ}$ and the forearm fully supinated. The patient actively and fully extends all the digits while the wrist is held in neutral and faces the examiner. The examiner places one hand to palpate the ECU tendon and with the other on the radial side of the adducted thumb. The patient is asked to resist the examiner's attempts to adduct the thumb and the examiner's other hand feels the ECU tendon contract isometrically to resist adduction of the thumb as this action is performed. Pain along the course of the ECU tendon during this manoeuvre has been shown to represent a positive test for ECU tendinosis.

ECU tendinosis can coexist with other conditions in the ulnar side of the wrist, so the diagnosis must not be considered as an exclusion of all other possible diagnoses. A full clinical and radiological assessment of the other important ulnar-sided wrist structures is mandatory to exclude coexistent pathology in the triangular fibrocartilage complex (TFCC), lunotriquetral ligament, distal radioulnar joint or ulnar styloid.

\section{Imaging}

In equivocal or difficult cases, ultrasound (US) or MRI are the imaging modalities of choice to supplement the clinical diagnosis of ECU tendinopathy and instability. Conventional X-rays are not routinely required.

US has several advantages. The examination is rapid, inflammatory changes can be assessed with Doppler imaging without the need for intravenous contrast agents, the opposite limb may be used for comparison and dynamic assessment can be performed between pronation and supination. MRI is able to assess other structures such as the TFCC that are not easily accessible to US evaluation.

\section{Normal ECU}

The ECU has a flattened ovoid configuration in transverse section at the level of the ulnar groove and lies deep to the subsheath (figure 5A). The ECU subsheath is inconsistently seen on MRI. ${ }^{12}$ As the tendon passes distal to the styloid process there is normal prominence of the hypoechoic synovial tendon sheath, which should not be mistaken for tenosynovitis. The extensor retinaculum is a thin structure around the periphery of ECU (figure 5B). It is hyperechoic on US (but has anisotropic properties) and low signal intensity (SI) on MRI. In supination the tendon demonstrates minor subluxation, lying towards the ulnar aspect of the groove (figure $5 \mathrm{C}$ ).

\section{Tenosynovitis}

Anechoic, easily compressible fluid surrounding the tendon is identified on US (high SI fluid on MRI). Vascularity on Doppler should be minimal or absent (figure 6). The underlying tendon may appear normal. Echogenic hypervascular tendon sheath thickening is more likely to be associated with inflammatory arthritis. $^{13}$

\section{Tendinopathy}

In early tendinopathy, the degree of tendon thickening may be subtle so comparison with the opposite side on US is helpful. As the disease progresses, the tendon thickening becomes more pronounced, there are poorly defined low echo areas within the tendon substance on US. ${ }^{13}$ Tendon neovascularisation may be present on Doppler imaging. On MRI, areas of tendonosis are manifest as areas of moderate increased SI on all pulse sequences. Care must be taken when assessing the MR appearances of the ECU as altered signal (but not thickening) can be seen in normal. This is thought to result either from MRI 'magic angle' artefact or the effect of the two functional bundles that are present in the distal ECU tendon. ${ }^{14}$

Partial tendon tears are seen as either clefts or 'splits' within the tendon substance (transverse images) or as areas of attenuation of tendon thickness (longitudinal images; figure 7).

Complete tendon rupture is uncommon. In chronic rupture there is tendon non-visualisation with proximal muscle atrophy and an absence of other soft tissue findings. In acute rupture the 
A

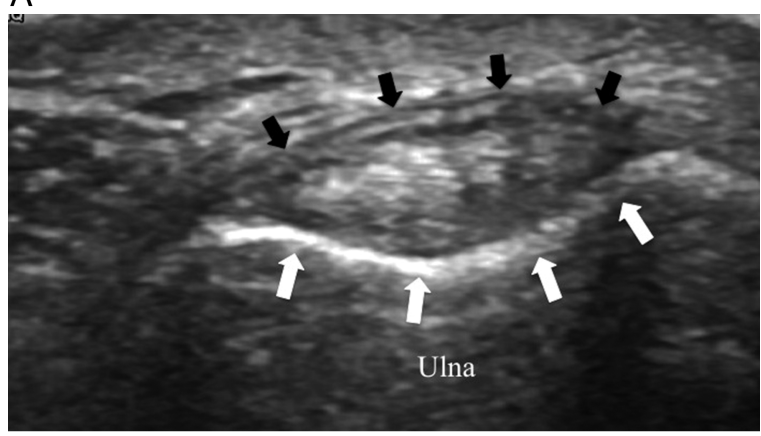

B

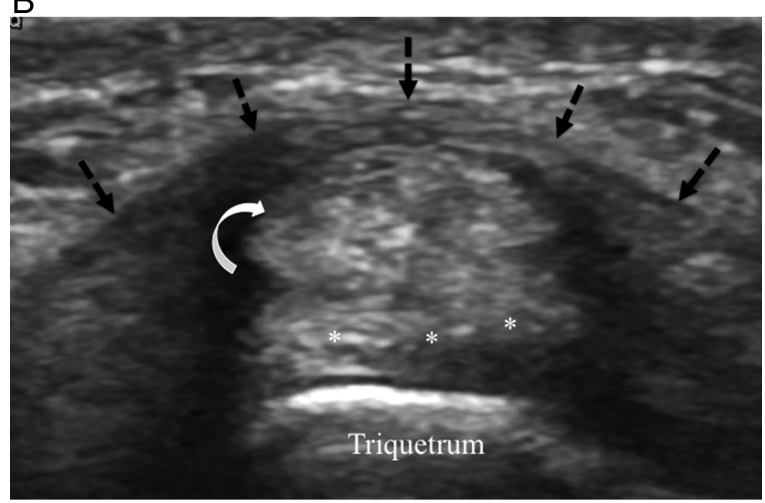

C

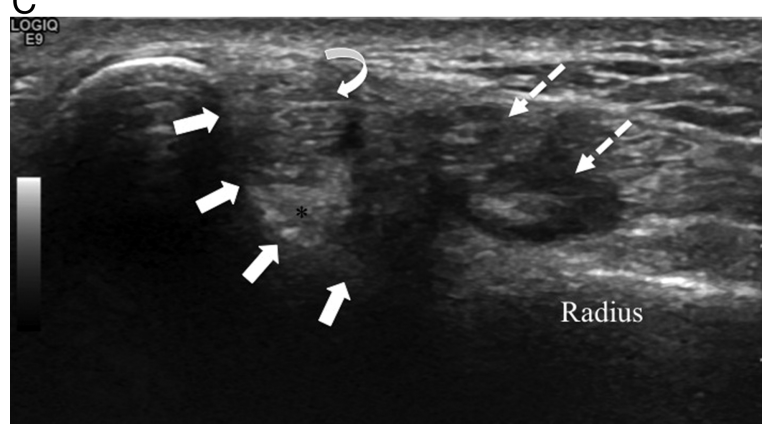

Figure 5 Axial ultrasound images of the normal extensor carpi ulnaris (ECU) tendon. In wrist pronation (A) the tendon lies within the ulnar groove (white arrows). The subsheath (black arrows) is immediately superficial to the tendon and attaches to the ulna. Distal to the ulna (B) the tendon (curved white arrow) lies superficial to the meniscal homologue of the triangular fibrocartilage complex (asterix) and the triquetrum. The extensor retinaculum (broken black arrows) displays hyper-reflective and hyporeflective properties due to the effects of anisotropy. In wrist supination (C) the tendon (curved white arrow) moves to the ulnar aspect of the groove (white arrows), and a small area of echo bright fatty tissue lies in the radial aspect of the groove (black asterisk). The tendons of the fourth and fifth compartments (broken white arrows) now lie in closer relation to the ECU tendon.

focal defect of the tendon is evident with varying degrees of retraction of the tendon end, with associated soft tissue haemorrhage.

\section{Tendon instability}

The ECU tendon displacement of up to $50 \%$ of the tendon width from the ulnar groove may be observed in asymptomatic patients, and is greatest in supination, flexion and ulnar deviation. $^{7}$ It may therefore be difficult to distinguish tendon subluxation that occurs following athletic subsheath injuries. Failure of the tendon to return to a normal position in pronation is
A

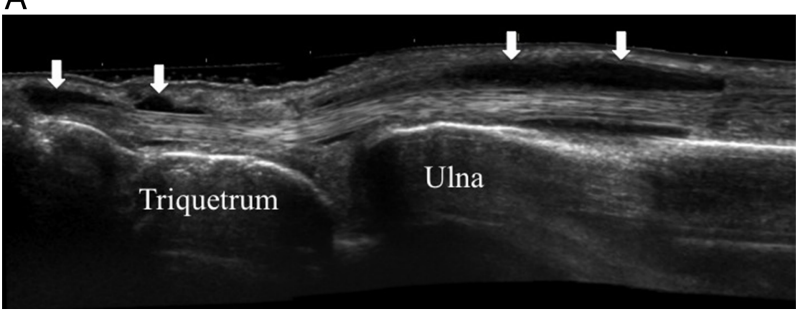

B

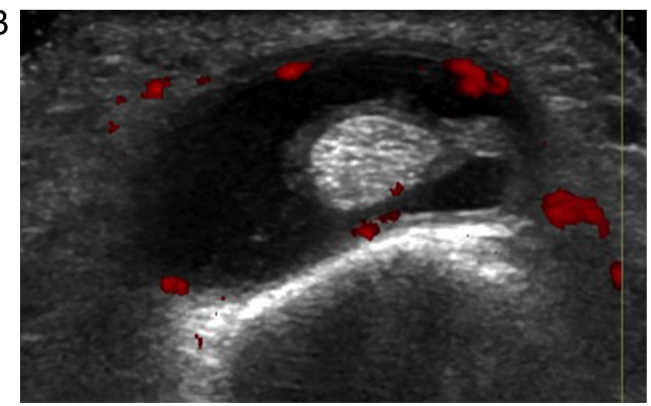

Figure 6 Longitudinal ultrasound image (A) of extensor carpi ulnaris tenosynovitis with prominent areas of anechoic fluid in the tendon sheath (white arrows). The tendon is normal. There is only minor inflammatory change in the tendon sheath on axial power Doppler imaging (B). (Reproduced from Imaging of Pain, Waldman SD \& Campbell RSD, Ch 129 Extensor Carpi Ulnaris, p330, Copyright 2011, with permission from Elsevier.)

uncommon. ${ }^{12}$ MRI does not invariably demonstrate the site of subsheath tear. Associated MRI signs of subsheath injuries include tendinopathy, tenosynovitis and marrow oedema in the head of the ulna. ${ }^{12}$ Acute rupture of the subsheath will be associated with oedema and haemorrhage surrounding the tendon, with or without tendon subluxation (figures 8 and 9).

\section{Management and outcomes}

ECU tendinosis

Acute tendinosis of the ECU usually responds to non-operative measures of rest, activity modification, splintage (in a position of $30^{\circ}$ wrist extension and ulnar deviation) or, occasionally,

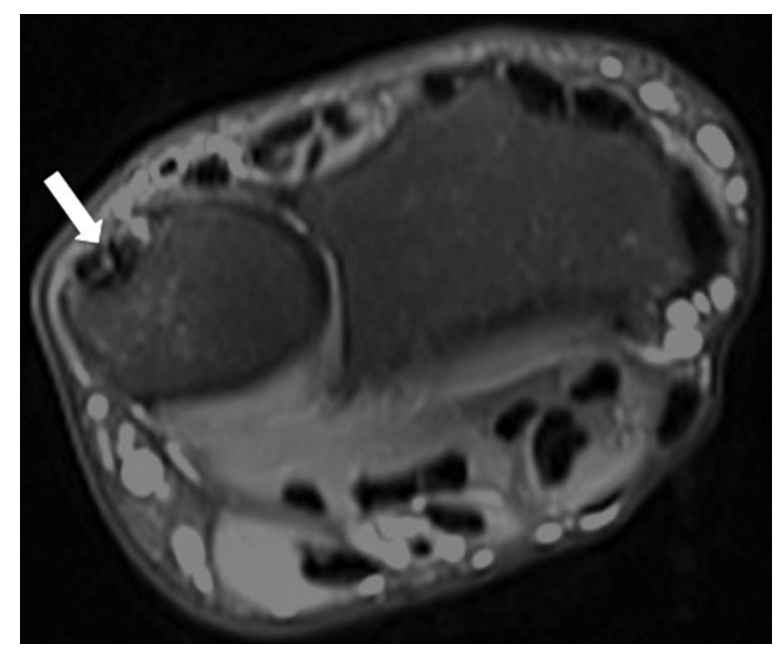

Figure 7 Axial fat saturated T2-weighted image of the wrist. There is a linear area of high signal intensity within the extensor carpi ulnaris tendon (white arrow) representing a longitudinal partial cleft tear. 


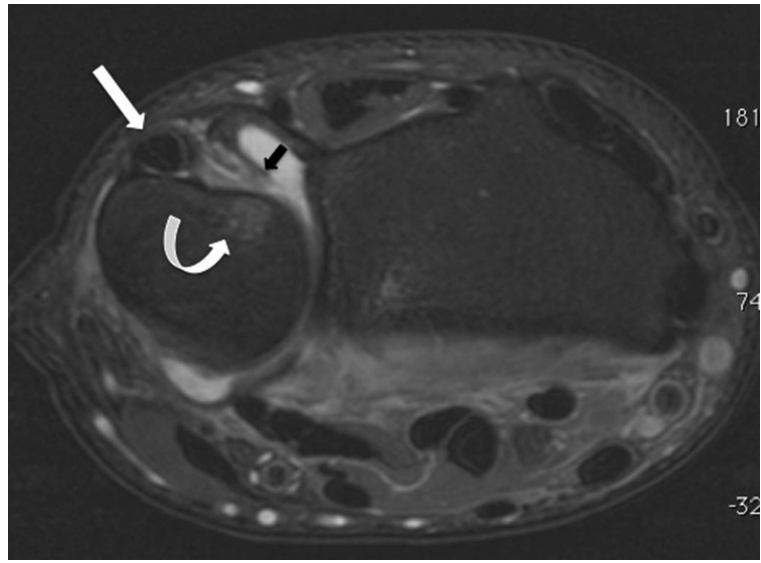

Figure 8 Axial T2 fat saturated MRI of the wrist in a rugby league player following an acute extensor carpi ulnaris subsheath injury. The tendon (white arrow) is subluxed in an ulnar direction and the subsheath is torn at its radial insertion on the ulna (black arrow). There is associated marrow oedema in the head of the ulna (curved white arrow). There were associated injuries including an acute triangular fibrocartilage tear, and there is an effusion in the distal radioulnar joint.

immobilisation in a short-arm plaster cast in the same position for a 3 -week period.

Some prefer to rest the wrist in a long-arm cast with the forearm in pronation, so that the ECU tendon sits comfortably located within the dorsal ulnar groove. There is no evidence to support one type of cast over another.

Rehabilitation strategies are based on the severity of tendinopathy. ${ }^{5}$ Treatment of the early reactive phase consists of load management and isometric exercises until the pain settles (typically over 5-10 days). Load can then be increased in stages. Ibuprofen is thought to be a helpful adjunct during this phase.

In chronic tendinopathy, without a sudden increase in pain, a combination of load management, eccentric work, isometrics and strength exercises are likely to help. Some of the changes within the tendon may be reversible but it is likely this is a condition that will need to be managed in the long term.

If symptoms are not relieved by non-operative measures an injection of steroid into the fibro-osseous sheath should be considered. A preliminary injection of local anaesthetic can be used for diagnostic confirmation and also as a mechanical hydrodissector to create space within the sheath for subsequent steroid injection.

Injections are best performed under US guidance; to ensure accurate placement of injectate and to avoid intratendinous injection (and risk of precipitating subsequent rupture).

In patients with persistent ECU tendinosis, cocompartment release should be considered. ${ }^{4}$ This involves division of the intercompartmental septum between the fifth and sixth extensor compartments, hence increasing the compartmental volume without threatening its stabilising function.

Return to athletic activity should be based on rehabilitation goals of range of motion and strength. These should ideally reach $80 \%$ of the uninjured side before returning to sports. ${ }^{15}$

\section{ECU instability}

Asymptomatic subluxation of the ECU tendon does not always require treatment. However, in cases of tendon instability associated with secondary tendinosis, conservative management of the tendinosis alone is unlikely to be successful.

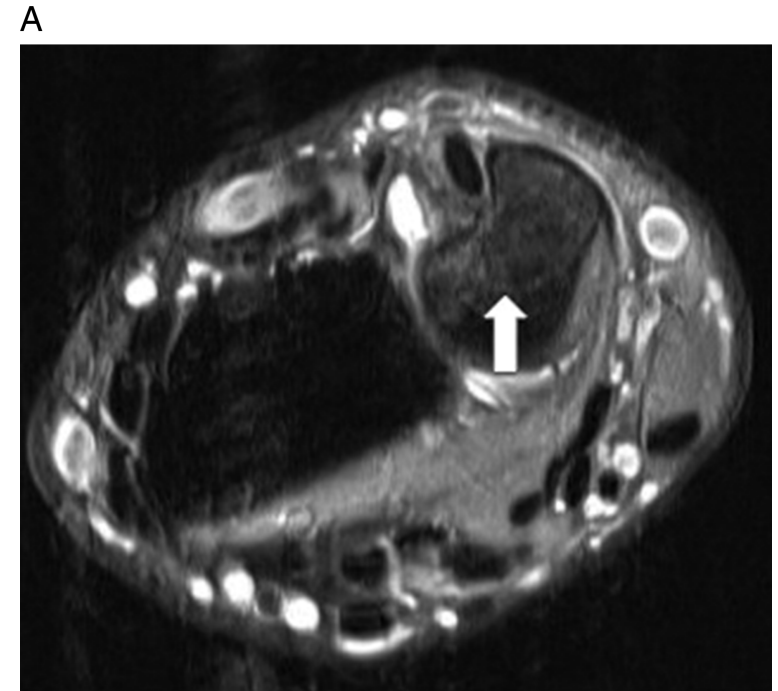

B

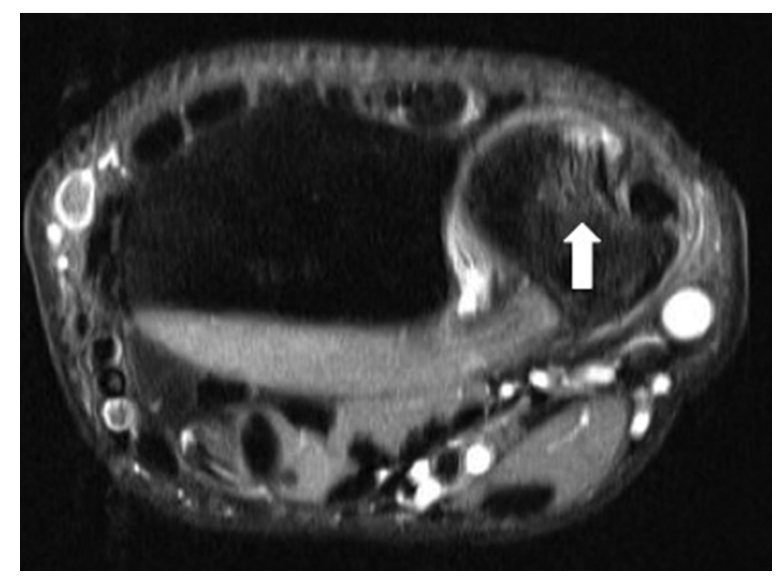

Figure 9 Axial fat saturated T2-weighted images of the wrist following a subsheath injury. In supination (A), the ECU tendon is subluxed and lies in the ulnar portion of the bony groove. This position is fixed and the tendon does not return to it normal position in pronation (B). There is also marrow oedema within the head of the ulna indicating the acute nature of the subsheath injury (white arrows).

Early diagnosis of an acute traumatic unstable ECU tendon may be managed by reduction of the subluxed tendon and immobilisation for a period of up to 6 weeks. Reduction is achieved by positioning the wrist in radial deviation and the forearm in pronation. ${ }^{16}$ The tendon will relocate into the ulnar groove and should then be maintained in this position by application of a long-arm cast. A report of 28 professional tennis players ${ }^{9}$ demonstrated successful outcome in all cases of tendon instability when treated with a prolonged period of immobilisation of up to 4 months. This prolonged immobilisation regime obviously has an impact on athletic conditioning and performance and may not be favoured in some circumstances.

In chronic subluxation surgical reconstruction of the sixth extensor compartment may be indicated, particularly in an elite athlete. Different techniques have been reported using a sling created from the remaining extensor retinaculum. ${ }^{17}{ }^{18}$ These type of reconstructions are termed 'non-anatomic' because they do not recreate the normal anatomy of the ECU complex. The repair must not be too tight to prevent smooth gliding of the newly stabilised tendon with wrist movement.

Anatomic reconstructions are indicated when the periosteum and tendon sheath strip off the distal ulna and the tendon 
subluxes within an expanded subsheath. The subsheath is reattachment on the ulnar groove with a series of small bone anchors. Twenty of 21 patients treated in this way, at an average of 17 months after injury, returned to their previous sport or employment with no reports of recurrent subluxation. ${ }^{19}$

\section{Summary}

The ECU tendon disease occurs commonly in certain sports such as golf, tennis and rugby. Many cases can be managed by conservative measures and rehabilitation. Distinction must be made between stable and unstable conditions. Tendon subluxation is a difficult condition to evaluate and may occur in asymptomatic patients. Management remains controversial.

Imaging with US or MRI is a useful supplement to clinical examination when there are atypical features and in cases that are resistant to conservative management. US-guided injection therapy is a useful adjunct for treatment of tenosynovitis in some cases.

Surgery is not often required, but may be indicated in cases of acute traumatic subsheath injuries and in cases of chronic subluxation combined with tendinopathy.

\section{What are the new findings}

- Extensor carpi ulnaris (ECU) problems are common in a diverse range of sports such as golf, rugby league and tennis.

- ECU instability can be constitutional and, if asymptomatic, does not require treatment

- A 3-T MRI is preferable (compared with 1.5 T) when imaging a wrist particularly as it shows detail of other structural failure in the differential diagnosis such as the triangular fibrocartilage and ligament injuries

- Rupture of the ECU is uncommon in a normal tendon but in tennis has been shown to occur after repeated steroid injections

Acknowledgements The members of the European Tour Advisory Board and, in particular, Mr Rob Hillman, the Tour Physiotherapy Director and the Physiotherapy and Sports Medical team of the European Tour for their comments, support and help with the study.

Contributors RH had the initial idea for the paper. The authors are from different specialities and have contributed according to their specialisation and the radiologists have provided illustrations of actual cases they have been involved with (and in most cases having been referred by RH and DC) and demonstrate the role of modern radiology. The paper was written in sections and split between the authors with RC as the editor. RH has liaised with the Journal to gauge the content. All have agreed on the final draft.
Funding Expenses paid by the European Tour.

Competing interests None.

Provenance and peer review Not commissioned; externally peer reviewed.

Open Access This is an Open Access article distributed in accordance with the Creative Commons Attribution Non Commercial (CC BY-NC 3.0) license, which permits others to distribute, remix, adapt, build upon this work non-commercially, and license their derivative works on different terms, provided the original work is properly cited and the use is non-commercial. See: http://creativecommons.org/ licenses/by-nc/3.0/

\section{REFERENCES}

1 Brigstocke $\mathrm{G}$, Hearnden $\mathrm{A}$, Holt CA, et al. The functional range of movement of the human wrist. J Hand Surg Eur Vo/2013;38:554-6.

2 Brigstocke $\mathrm{GH}$, Hearnden $\mathrm{A}$, Holt $\mathrm{C}$, et al. In-vivo confirmation of the use of the dart thrower's motion during activities of daily living. J Hand Surg Eur Vol 2012 [Epub ahead of print].

3 Horii $\mathrm{E}, \mathrm{An} \mathrm{KN}$, Linscheid RL. Excursion of prime wrist tendons. J Hand Surg [Am] 1993; 18:83-90.

4 Hajj AA, Wood MB. Stenosing tenosynovitis of the extensor carpi ulnaris. J Hand Surg [Am] 1986;11:519-20.

5 Cook JL, Purdam CR. Is tendon pathology a continuum? A pathology model to explain the clinical presentation of load-induced tendinopathy. Br J Sports Med 2009:43:409-16.

6 Geissler WB. Carpal fractures in athletes. Clin Sports Med 2001;20:167-88.

7 Lee KS, Ablove RH, Singh $\mathrm{S}$, et al. Ultrasound imaging of normal displacement of the extensor carpi ulnaris tendon within the ulnar groove in 12 forearm-wrist positions. AJR Am J Roentgenol 2009;193:651-5.

8 Rettig AC, Ryan RO, Stone JA. Epidemiology of hand injuries in sports. In: Strickland JW, Rettig AC. eds Hand injuries in athletes. PA: WB Saunders, 1992:37-449.

9 Montalvan B, Parier J, Brasseur JL, et al. Extensor carpi ulnaris injuries in tennis players: a study of 28 cases. Br J Sports Med 2006:40:424-9; discussion 429.

10 Batt ME. A survey of golf injuries in amateur golfers. Br J Sports Med 1992:26:63-5.

11 Ruland RT, Hogan CJ. The ECU synergy test: an aid to diagnose ECU tendonitis J Hand Surg [Am] 2008;33:1777-82.

12 Jeantroux J, Becce $F$, Guerini $H$, et al. Athletic injuries of the extensor carpi ulnaris subsheath: MRI findings and utility of gadolinium-enhanced fat-saturated T1-weighted sequences with wrist pronation and supination. Eur Radiol 2011;21:160-6

13 Bianchi S, Wrist MC. In: Bianchi S, Matrtinoli C. eds Ultrasound of the musculoskeletal system. Berlin, Heidelberg: Springer-Verlag, 2007:425-94.

14 Timins ME, O'Connell SE, Erickson SJ, et al. MR imaging of the wrist: normal findings that may simulate disease. Radiographics 1996;16:987-95.

15 Topper SM, Wood MB, Cooney WP. Athletic injuries of the wrist. In: Cooney WP, Linscheid RL, Dobyns JH, eds. The wrist: diagnosis and operative treatment. St Louis: Mosby, 1998:1031-74.

16 Patterson SM, Picconatto WJ, Alexander JA, et al. Conservative treatment of an acute traumatic extensor carpi ulnaris tendon subluxation in a collegiate basketball player: a case report. J Athl Train 2011;46:574-6.

17 Burkhart SS, Wood MB, Linscheid RL. Posttraumatic recurrent subluxation of the extensor carpi ulnaris tendon. J Hand Surg [Am] 1982:7:1-3.

18 Eckhardt WA, Palmer AK. Recurrent dislocation of extensor carpi ulnaris tendon. J Hand Surg [Am] 1981;6:629-31.

19 MacLennan AJ, Nemechek NM, Waitayawinyu T, et al. Diagnosis and anatomic reconstruction of extensor carpi ulnaris subluxation. J Hand Surg [Am] 2008:33:59-64. 